\title{
Deregulated WWOX is involved in a negative feedback loop with microRNA-214-3p in osteosarcoma
}

\author{
KAITUO GAO, JIJUAN YIN and JIAN DONG \\ Department of Orthopedics, Linyi People's Hospital, Linyi, Shandong 276003, P.R. China
}

Received December 29, 2015; Accepted October 31, 2016

DOI: $10.3892 / \mathrm{ijmm} .2016 .2800$

\begin{abstract}
WW domain-containing oxidoreductase (WWOX) is frequently inactivated in human osteosarcoma, and the restoration of its expression can suppress tumorigenicity in WWOX-negative OS cells. However, its regulatory mechanisms remain to be fully elucidated. In the present study, we demonstrate that WWOX is downregulated and that it regulates proliferation and epithelial-to-mesenchymal transition (EMT)associated protein expression in osteosarcoma. As shown by our results, WWOX overexpression by transfection with WWOX overexpression plasmids suppressed the proliferation, migration and invasion of osteosarcoma MG63 cells (as shown by MTT and migration and invasion assays). The silencing of microRNA (miR)-214-3p by transfection with anti-miR-14-3p upregulated WWOX protein expression and also inhibited the proliferation, migration and invasion of osteosarcoma cells. Additionally, we found that WWOX negatively regulated miR-214-3p and miR-10b expression. Our findings define a negative feedback pathway in control of WWOX and miR-214-3p expression, thus providing novel molecular targets for the treatment of osteosarcoma.
\end{abstract}

\section{Introduction}

Osteosarcoma is the most common type of malignant bone tumor, and it frequently originates in the metaphysis of the long bones (1). The prognosis of patients with osteosarcoma remains poor, as approximately $80 \%$ of patients eventually develop metastatic disease following surgical treatment (2) and pulmonary metastasis is the major cause of fatal outcome (3). The further elucidatation of the molecular mechanisms responsible for the development of osteosarcoma will not only help us to understand the pathogenesis and progression of the disease, but may also provided novel targets for effective therapies.

Correspondence to: Dr Jian Dong, Department of Orthopedics, Linyi People's Hospital, 48 Jiefang Road, Lanshan, Linyi, Shandong 276003, P.R. China

E-mail: dongjianly@163.com

Key words: osteosarcoma, WW domain-containing oxidoreductase, microRNA-214-3p
WW domain-containing oxidoreductase (WWOX; also known as FOR/WOX1) encodes a $46-\mathrm{kDa}$ protein with a short-chain dehydrogenase/reductase domain and two WW domains (4-7). The gene is altered through deletions or translocations in many types of cancer, including breast, prostate, esophageal, lung, stomach and pancreatic cancer (5,7-12). Moreover, WWOX protein is decreased or lost in the majority of these cancers, suggesting that the deregulation of WWOX expression may be involved in cancer development $(13,14)$.

The ectopic overexpression of WWOX in cancer cells lacking endogenous WWOX has been shown to lead to marked growth inhibition and to prevent the progression of tumors in nude mice $(15,16)$. The restoration of WWOX expression in cancer cells has been shown to contribute to caspase-mediated apoptosis (16) and decreased WWOX expression is associated with drug resistance (17). WWOX plays a critical role in determining the aggressive phenotype of osteosarcoma, and it had been suggested that the restoration of its expression may be an attractive therapeutic strategy (18). However, the regulatory mechanisms of WWOX remain to be fully elucidated in osteosarcoma.

MicroRNAs (miRNAs/miRs) are small non-coding RNAs, 20-22 nucleotides in length that have been shown to be involved in various types of cancer (19-21). miR-214-3p has been shown to be upregulated and to function as an oncogene in human ovarian cancer (22), gastric cancer (23), pancreatic cancer (24) and osteosarcoma (25). In addition, the upregulated expression of miRNA-214 is linked to tumor progression and adverse prognosis in pediatric osteosarcoma (25), and it has been shown to promote the proliferation and invasion of osteosarcoma cells through the direct suppression of leucine zipper, putative tumor suppressor 1 (LZTS1) (26).

In the present study, we aimed to elucidate the mechanisms of action of WWOX in osteosarcoma. We also aimed to determine the role of miRNA-214 in the development and progression of osteosarcoma. In addition, we wished to determine the existence of an association between WWOX and miRNA-214 in osteosarcoma in an effort to provide more efficient therapeutic strategies for the treatment of osteosarcoma and to prevent tumor metastasis.

\section{Materials and methods}

Tissue samples and cell lines. Osteosarcoma tissues and adjacent normal tissues $(n=6)$ were collected from patients at surgery 
at Linyi People's Hospital, Linyi, China. The Medical Ethics Committee of Linyi People's Hospital approved the study, and all patients agreed to participate in the project and provided written informed consent prior to obtaining the samples. The human osteosarcoma cell line, MG63, was purchased from the Cell Bank of the Chinese Academy of Sciences (Beijing, China). The cells were maintained in RPMI-1640 medium (HyClone Co., Logan, UT, USA) supplemented with $10 \%$ fetal bovine serum and $1 \%$ penicillin $\mathrm{G} /$ streptomycin.

Cell transfection. All WWOX overexpression plasmids were purchased from Tiangen (Tianjin, China). The empty vectors were also obtained from Tiangen. Anti-miR-214-3p/scramble inhibitor was purchased from Ambion, Inc. (Austin, TX, USA). The cells were seeded into 6 -well plates $24 \mathrm{~h}$ prior to transfection. When the MG63 cells reached $80 \%$ confluence, the expression plasmids were transfected into the cells using Lipofectamine 2000 (Invitrogen, Carlsbad, CA, USA) according to manufacturer's instructions.

Western blot analysis. The cells were collected and lysed using RIPA lysis buffer. Proteins were separated by sodium dodecyl sulfate polyacrylamide gel electrophoresis (SDS-PAGE) and then electroplotted onto polyvinylidene difluoride (PVDF) membranes. The blots were blocked and incubated with antibodies against WWOX (ab137726), cyclin D1 (ab134175), p53 (ab62376), p21 (ab109520), p27 (ab32034), c-myc (ab32072), E-cadherin (E-Cad; ab15148), zinc finger E-box-binding homeobox 1 (ZEB1; ab203829), vimentin (ab16700), Slug (ab27568) and $\beta$-actin (ab8227) (all from Abcam, Cambridge, MA, USA) overnight $4^{\circ} \mathrm{C}$. After washing, the membranes were incubated at $37^{\circ} \mathrm{C}$ for $1 \mathrm{~h}$ with goat anti-rabbit IgG H\&L (HRP) secondary antibody (ab6721; Abcam) and visualized using ECL detection reagent.

MTT cell proliferation assay. Following transfection, the cells were re-seeded at a density of $2 \times 10^{4}$ cells/well in 96-well plates containing $0.2 \mathrm{ml}$ RPMI-1640 (with 10\% FBS). Subsequently, $20 \mu \mathrm{l}$ of 3-(4,5-dimethylthiazol-2-yl)-2,5-diphenyltetrazolium bromide (MTT) were added followed by incubation for a further $4 \mathrm{~h}$ at $37^{\circ} \mathrm{C}$. A total of $150 \mu \mathrm{l}$ of dimethyl sulfoxide was then added to each well and the absorbance was measured at $570 \mathrm{~nm}$ on an enzyme immunoassay analyzer (Bio-Rad, Hercules, CA, USA).

BrdU assay. Cell proliferation was also assessed using a colorimetric BrdU proliferation kit by following the manufacturer's instructions (Roche; Cat. no. 11647229001). Briefly, the cells treated with the peptides were labeled with BrdU for 3-4 h. The genomic DNA was then fixed and denatured, then incubated with the peroxidase-conjugated anti-BrdU antibody for $90 \mathrm{~min}$. The substrate of the conjugated peroxidase was then added and the reaction product was measured by the absorbance $\left(\mathrm{A}_{370 \mathrm{~nm}}-\mathrm{A}_{492 \mathrm{~nm}}\right)$. The results were then normalized by the number of total viable cells, which was determined by a side-by-side cell viability assay as described above.

Migration and invasion assays. Following transfection, the cells were added to the chamber with the non-coated membrane (BD Biosciences, San Diego, CA, USA), and cells migrating to the lower sides of the filters were fixed in $4 \%$ paraformaldehyde and stained with crystal violet. Cell invasion assay was performed as the migration assay except that the chamber was coated with Matrigel (BD Biosciences) and the cells were observed under a microscope.

Reverse transcription-quantitative polymerase chain reaction (RT-qPCR). Total RNA was extracted from the cells using TRIzol reagent (Invitrogen) according to manufacturer's instructions. The First-Strand cDNA was generated by reverse transcription with random hexamer primers and a M-MLV reverse transcriptase kit (Promega, Madison, WI, USA). Quantitative PCR (qPCR) for WWOX was carried out using Power SYBR-Green PCR Master Mix (Applied Biosystems, Carlsbad, CA, USA) according to the manufacturer's instructions. We used 5'-AGTTCCTGAGCGAGTGGACC-3' as the forward primer and 5'-TTACTTTCAAACAGGCC ACCAC-3' as the reverse primer. GAPDH was used as a loading control. The primer sequences for GAPDH were as follows: forward, 5'-ACCACAGTCCATGCCATCAC-3' and reverse, 5'-TCCACCACCCTGTTGCTGTA-3'. The thermal cycle profile was as follows: denaturation for $30 \mathrm{sec}$ at $95^{\circ} \mathrm{C}$, annealing for $45 \mathrm{sec}$ at $52-58^{\circ} \mathrm{C}$ depending on the primers used, and extension for $45 \mathrm{sec}$ at $72^{\circ} \mathrm{C}$. qPCR for miR-214-3p was performed using the mirVana qRT-PCR miRNA detection kit and qRT-PCR Primer Sets, according to the manufacturer's instructions (Ambion, Inc.).

Immunofluorescence assay. The cells grown on polylysinetreated slides were washed with phosphate-buffered saline (PBS) and fixed with $4 \%$ paraformaldehyde on ice, and then blocked with 5\% BSA at room temperature. Anti-WWOX antibody (ab137726; Abcam) were added at $4^{\circ} \mathrm{C}$ overnight followed by incubation with goat anti-rabbit (Alexa Fluor ${ }^{\circledR} 488$ ) secondary antibody (ab150077; Abcam) at room temperature for $30 \mathrm{~min}$. The samples were then washed 3 times with PBS-Tween-20, and the nuclei were stained with 4,6-diamidino2-phenylindole (DAPI).

miRNA microarray analysis. Microarray analysis was performed using Affymetrix GeneChip miRNA 3.0 arrays kit (Affymetrix, Santa Clara, CA, USA) according to the manufacturer's instructions. After washing and staining, the arrays were scanned using GeneChip scanner 3000 (Affymetrix).

Northern blot analysis. For northern blot analysis of the cells, all RNA samples were prepared and separated in a denaturing urea-polyacrylamid gel and transferred to nylon membranes by semi-dry electroblotting. The RNA probes were labeled with $\left[\gamma^{32} \mathrm{P}\right]$ ATP complementary to the target miRNAs. U6 was used as a loading control.

Bioinformatics analysis. The analysis of potential microRNA target site using the commonly used prediction algorithms miRanda (http://www.microrna.org/).

Statistical analysis. Data are expressed as the means \pm SD, from at least 3 separate experiments and were analyzed using one-way ANOVA or the Student's t-test. A value of $\mathrm{p}<0.05$ was considered to indicate a statistically significant difference. 


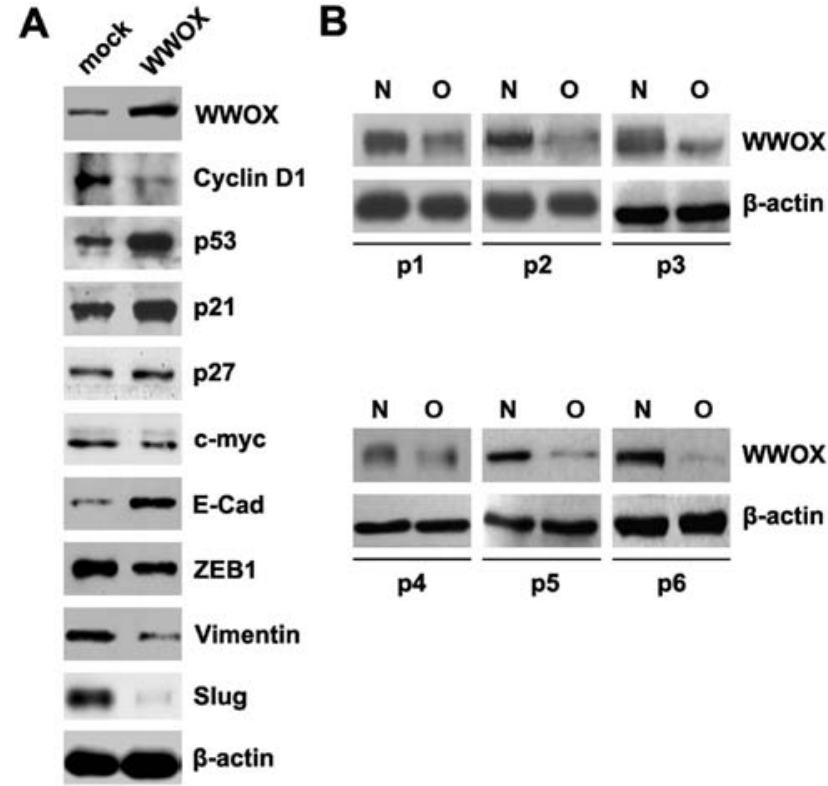

Figure 1. WWOX regulates cell proliferation and EMT-associated protein expression, and its expression is downregulated in osteosarcoma. (A) Western blot analyssi of WWOX, cyclin D1, p53, p21, p27, c-myc, E-Cad, ZEB1, vimentin and Slug in MG63 cells transfected with WWOX overexpression plasmids or empty vectors (mock). $\beta$-actin was used as a loading control. $\mathrm{n}=3$ experiments. (B) Western blot analysis of WWOX protein expression in osteosarcoma tissues $(\mathrm{O})$ and adjacent normal tissues $(\mathrm{N})$. $\beta$-actin was used as a loading control. $n=6$ samples.
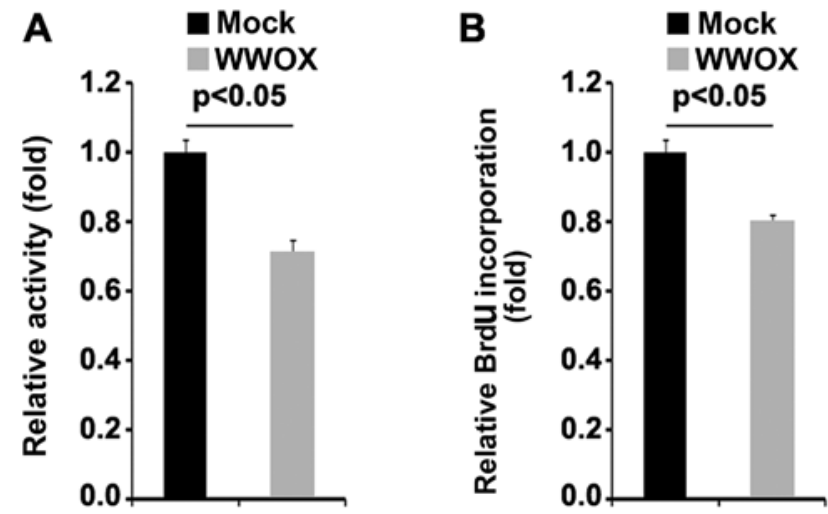

Figure 2. WWOX inhibits the proliferation of MG63 osteosarcoma cells (A) MTT assay for the proliferation of MG63 cells. MG63 cells were transfected with WWOX overexpression plasmids or empty vectors (mock) and cell proliferation was then measured by MTT assay. $n=3$ experiments. (B) BrdU incorporation assay for MG63 cell proliferation. Cells were transfected with WWOX overexpression plasmids or empty vectors (mock). $n=3$ experiments.

\section{Results}

WWOX regulates cell proliferation and epithelial to mesenchymal transition (EMT)-associated gene expression and its expression is downregulated in osteosarcoma. In an attempt to determine the role of WWOX in regulating cell proliferation and EMT in osteosarcoma, the MG63 cells were transfected with WWOX overexpression plasmids or empty vectors. Following stable transfection, WWOX expression was detected by western blot analysis and 9 proliferation- and EMT-associated markers were also examined by western blot
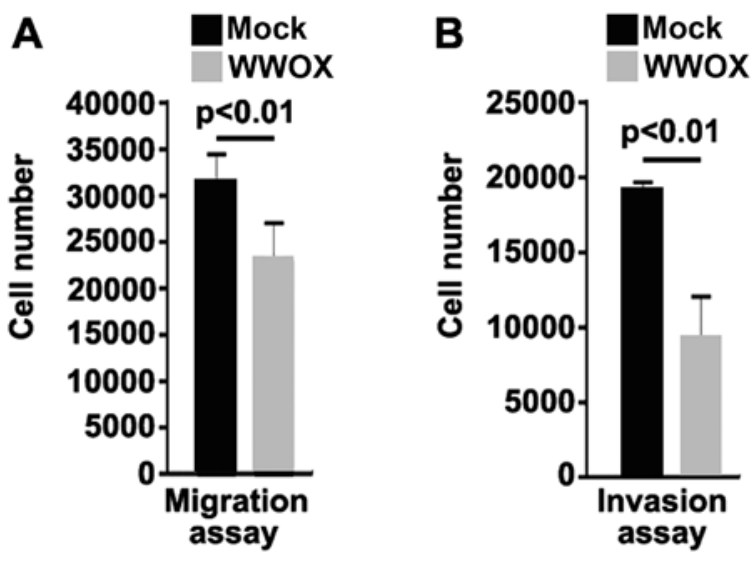

Figure 3. WWOX inhibits the migration and invasion of MG63 osteosarcoma cells. (A) Matrigel invasion assay of MG63 cells transfected with WWOX overexpression plasmids or empty vectors (mock). $n=3$ experiments. (B) Transwell invasion assay of MG63 cells transfected with WWOX overexpression plasmids or empty vectors (mock). $n=3$ experiments.

analysis in the MG63 cells. The results revealed that transfection with WWOX overexpression plasmids markedly increased WWOX protein expression. It also promoted p53, p21 and E-Cad expression and suppressed cyclin D1, ZEB1, vimentin and Slug expression in the MG63 cells (Fig. 1A). To assess the expression of WWOX in osteosarcoma, western blot analysis was conducted on 6 pairs of osteosarcoma tissues and matched adjacent normal tissue samples. The expression of WWOX was consistently lower in the osteosarcoma tissues compared with the normal tissues (Fig. 1B).

WWOX inhibits the proliferation of MG63 osteosarcoma cells. In order to determine the role of WWOX in regulating the proliferation of MG63 cells, the proliferation rates of MG63 cells transfected with WWOX overexpression plasmids or empty vectors were examined by MTT assay. The results revealed that the overexpression of WWOX significantly suppressed the proliferation of MG63 cells (Fig. 2A). This was confirmed by BrdU incorporation assay which revealed that transfection with WWOX overexpression plasmids resulted in decreased DNA synthesis per viable MG63 cell (Fig. 2B).

WWOX inhibits the migration and invasion of osteosarcoma MG63 cells. E-Cad, ZEB1, Vimentin and slug are EMT associated markers and they are associated with invasion and migration in cancer (27-31). Given that WWOX markedly inhibited the expression of EMT-associated markers and that of markers associated with invasion and migration $(32,33)$, we then sought to determine whether WWOX has an effect on the migration and invasion of MG63 cells. The results from migration and invasion assay indicated that the overexpression of WWOX not only inhibited the migration of the MG63 cells, but also suppressed the invasion of these cells (Fig. 3).

Silencing of miR-214-3p upregulates WWOX protein expression in MG63 osteosarcoma cells. Having demonstrated that WWOX suppresses EMT, as well as the proliferation, migration and invasion of osteosarcoma cells, we then wished to determine the mechanisms regulating WWOX expression in 
A

Position 483-489 of WWOX 3'UTR

5'...AUUGUACUUAAACCUCCUGCUGU...

3' UGACGgacAGacACGgacGaCA

hsa-miR-214-3p
B

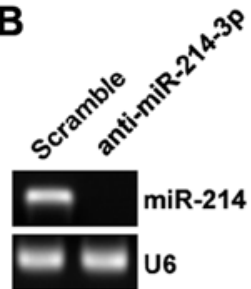

C

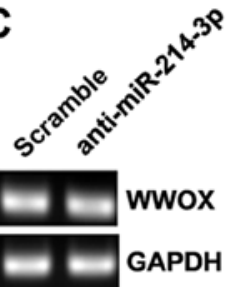

D

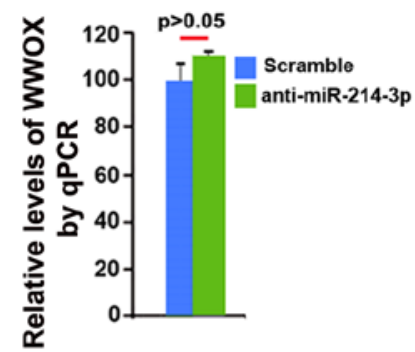

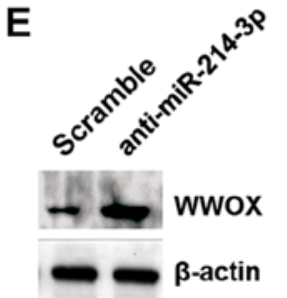

$F$
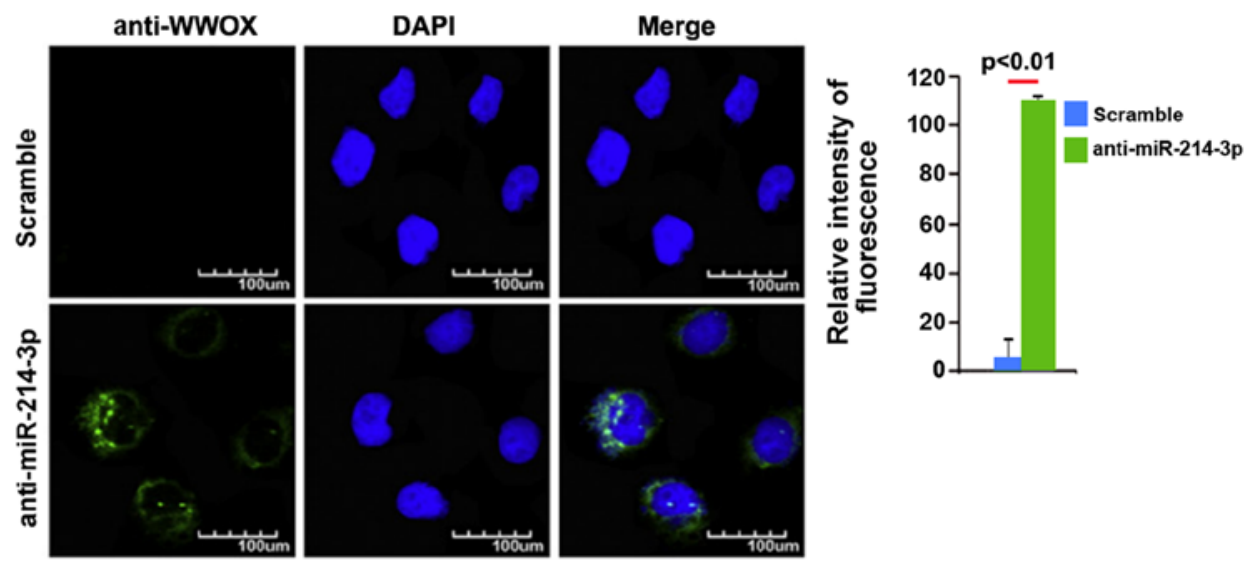

Figure 4. Silencing of miR-214-3p upregulates WWOX protein expression in MG63 osteosarcoma cells. (A) Schematic of predicted miR-214-3p-binding sites in the 3'UTR of WWOX mRNA by miRanda. (B) RT-qPCR for miR-214-3p in MG63 cells transfected with anti-miR-214-3p or scramble inhibitor. U6 was used as a loading control. $n=3$ experiments. (C) qPCR for WWOX in MG63 cells. MG63 cells were transfected with anti-miR-214-3p or scramble inhibitor. GAPDH was used as a loading control. $n=3$ experiments. (D) qPCR for WWOX in MG63 cells. MG63 cells were transfected with anti-miR-214-3p or scramble inhibitor. GAPDH was used a loading control. $n=3$. (E) Western blot analysis for WWOX protein expression in MG63 cells infected as indicated. $\beta$-actin was used as a loading control. $\mathrm{n}=3$ experiments. (F) Immunofluorescence assay for MG63 cells transfected as indicated. Left panel shows microscopic images of immunofluorescence staining of 1 representative experiment (x100 magnification). Right panel shows a graphic presentation of the mean fluorescence intensities. $n=3$ experiments.
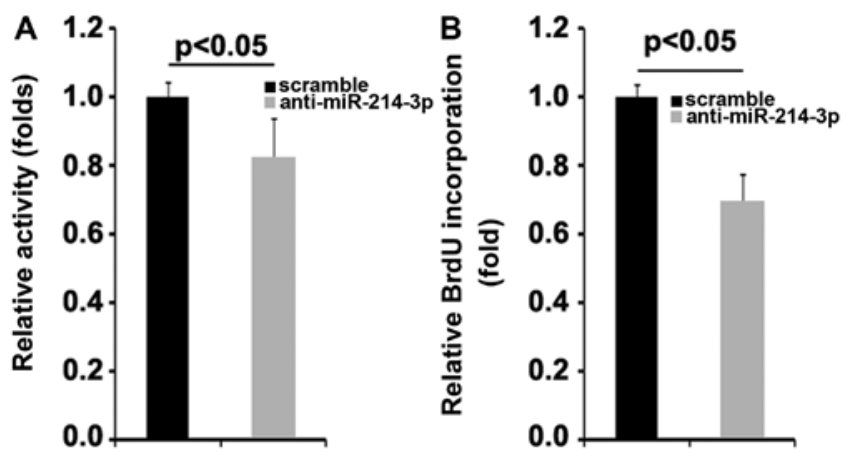

Figure 5. Silencing miR-214-3p inhibits the proliferation of MG63 osteosarcoma cells. (A) MTT assay for MG63 cell proliferation. MG63 cells were transfected as indicated and cell proliferation was then measured by MTT assay. $n=3$ experiments. (B) BrdU incorporation assay for MG63 cell proliferation. Cells were transfected as indicated. $n=3$ experiments.

the disease. miRNAs are a class of small non-coding RNAs (approximately 22 nucleotides in lenght) that negatively regulate protein-coding gene expression by targeting mRNA degradation or translation inhibition $(34,35)$. To further confirm whether WWOX is regulated by miRNAs, we used the commonly used prediction algorithm, miRanda (http://www.microrna.org/ microrna/home.do), to analyze the 3'UTR of WWOX. A dozen miRNAs were found by the algorithm. However, we were interested in miR-214-3p, as the expression of miR-214-3p has been confirmed to be significantly higher in osteosarcoma tissues than in normal tissues (25). It seems to play an important role in osteosarcoma. The target sites on the 3'UTR of WWOX are
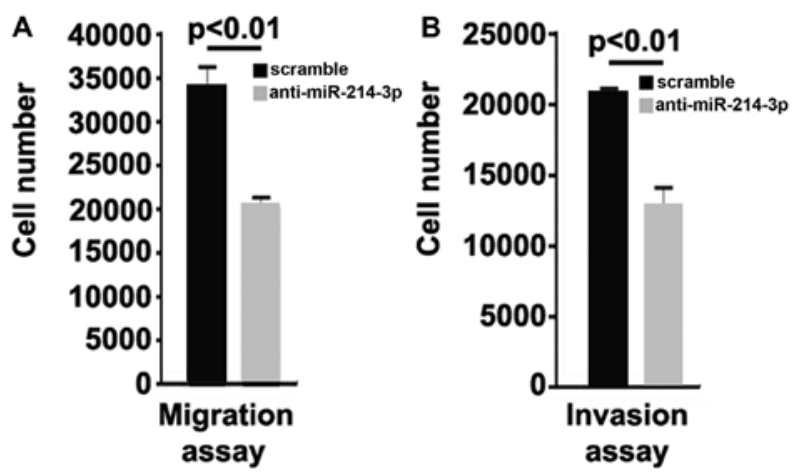

Figure 6. Silencing of miR-214-3p inhibits the migration and invasion of MG63 osteosarcoma cells. (A) Matrigel invasion assay for MG63 cells transfected as indicated. $n=3$ experiments. (B) Transwell invasion assay for MG63 cells transfected as indicated. $n=3$ experiments.

shown in Fig. 4A. We reasoned that miR-214-3p downregulates WWOX expression by targeting its 3'UTR and that WWOX protein is suppressed due to the overexpression of miR-214-3p in osteosarcoma. In an attempt to determine the role of miR-214-3p in regulating WWOX expression in osteosarcoma cells, the MG63 cells were transfected with anti-miR-214-3p and scramble inhibitor. Following transfection, miR-214-3p expression was detected by qPCR and the results revealed that miR-214-3p expression was significantly decreased by transfection of the cells with anti-miR-214-3p (Fig. 4B). We then performed RT-qPCR to detect WWOX mRNA expression in the MG63 cells transfected with anti-miR-214-3p or scramble inhibitor. 
A

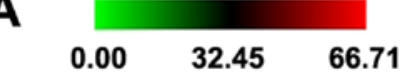

B

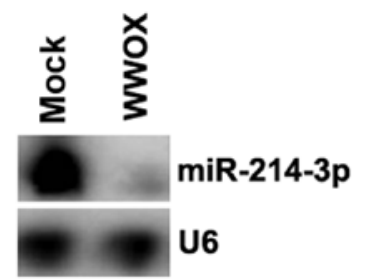

C

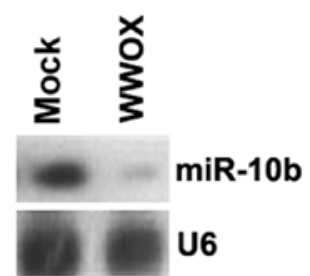

hsa-miR-214-3p

hsa-miR-15a

hsa-miR-101

hsa-miR-10b

hsa-miR-9

hsa-miR-373

hsa-miR-25

hsa-miR-196a

hsa-miR-222

Mock $\overline{\text { wwOX }}$

D

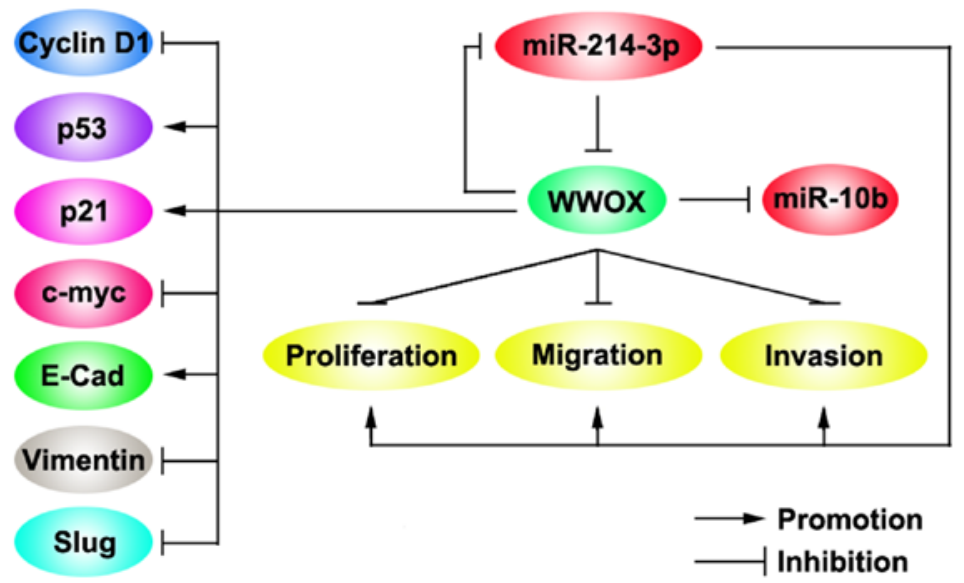

Figure 7. WWOX overexpression inhibits miR-214-3p and miR-10b expression in MG63 osteosarcoma cells. (A) miRNA microarray analysis for microRNAs in MG63 cells transfected with WWOX overexpression plasmids or empty vectors (mock). $n=3$ experiments. (B) Northern blot analysis for miR-213-3p in MG63 cells transfected with WWOX overexpression plasmids or empty vectors (mock). U6 was used as a loading control. $n=3$ experiments. (C) Northern blot analysis for miR-10b in MG63 cells transfected with WWOX overexpression plasmids or empty vectors (mock). U6 was used as a loading control. $\mathrm{n}=3$ experiments. (D) Diagram showin the mechanisms through which deregulated microRNA-214-3p is involved in a negative feedback loop with WWOX in osteosarcoma.

The results demonstrated that WWOX mRNA expression was not affected by transfection with anti-miR-214-3p compared with the scramble inhibitor-transfected groups (Fig. 4C and D). Moreover, western blot analysis was performed to detect WWOX protein expression in the MG63 cells transfected with anti-miR-214-3p or scramble inhibitor. The results revdealed that WWOX protein (Fig. 4E) was significantly upregulated in the cells after the silencing of miR-214-3p. Consistent with the results of western blot analysis, we performed immunofluorescence assay of the MG63 cells transfected with anti-miR-214-3p or scramble inhibitor. The results revealed that WWOX protein expression was evidently upregulated in the cells transfected with anti-miR-214-3p (Fig. 4F). These data demonstrated that WWOX protein expression was suppressed by the overexpression of miR-214-3p in osteosarcoma cells.

Silencing of miR-214-3p inhibits the proliferation of MG63 osteosarcoma cells. To determine the role of miR-214-3p in regulating the proliferation of MG63 cells, the proliferation rates of the MG63 cells were examined by MTT assay. The results demonstrated that the silencing of miR-214-3p significantly suppressed the proliferation of the MG63 cells (Fig. 5A). This was further confirmed by BrdU incorporation assay, which indicated that transfection with anti-miR-214-3p resulted in decreased DNA synthesis per viable MG63 cell (Fig. 5B).
Silencing of miR-214-3p inhibits the migration and invasion of MG63 osteosarcoma cells. We then sought to determine whether the silencing of miR-214-3p affects the migration and invasion of MG63 cells. The results of migration and invasion assay indicated that the silencing of miR-214-3p inhibited the migration and invasion of MG63 cells (Fig. 6).

WWOX inhibits miR-214-3p and miR-10b expression in osteosarcoma MG63 cells. Tumor suppressor genes can exert their effects by regulating miRNA expression in cancer (36) and miRNAs are known to play a role in cancer pathogenesis and some of them function as tumor suppressor genes or oncogenes (37-39). Thus, we hypothesized that WWOX functions as tumor suppressor by regulating relevant miRNAs. Thus miRNA microarrays was performed. RNAs isolated from the MG63 cells were hybridized to a custom miRNA microarray platform. Following hybridization, quantification, and normalization 3 times, a dozen miRNAs were foun to be altered in the MG63 cells. However, we were interested in miR-214-3p and miR-10b, as miR-214-3p and miR-10b appear to play the roles of oncogenes in various types of cancer $(22,25,40-43)$. The results of microarray analysis demonstrated that miR-214-3p and miR-10b expression was decreased $>50$-fold in the cells transfected with the WWOX overexpression plasmids (Fig. 7A). To further confirm the regulatory role of WWOX, we performed 
northern blot analysis to detect the expression of miR-214-3p and miR-10b. Consistent with the findings of microarray analysis, the results of northern blot analysis revealed that the overexpression of WWOX significantly downregulated miR-214-3p (Fig. 7B) and miR-10b (Fig. 7C) expression in the MG63 cells.

\section{Discussion}

Much research has been carried out in order to understand the pathogenesis of osteosarcoma and to provide novel therapeutic targets for the disease (44-46). miRNAs are small non-coding RNAs of 20-22 nucleotides in lenght that have been implicated in the development of various types of cancer (47). miR-214-3p expression is deregulated in several human tumors, including melanoma (48), breast cancer (49), ovarian cancer (22), gastric cancer (40), hepatocellular carcinoma (41) and osteosarcoma (25). Its pleiotropic and tumor-specific role in the formation and progression of various types of cancer is achieved by targeting its several target genes $(22,25,40,41,48,49)$. miR-214-3p can function as a key and core regulator by regulating vital signaling networks, such as $\beta$-catenin, PTEN/AKT and the tyrosine kinase receptor pathways $(42,43)$. Moreover, miR-214-3p can also regulate the expression of essential cancer-associated genes, for example, enhancer of zeste homolog 2 (Ezh2; the epigenetic repressor), p53, transcription factor AP-2 alpha (TFAP2) and miR-148b $(22,48,50)$. Consistent with its role of regulating essential cancer-associated genes, miR-214 seems to play crucial roles in promoting tumor cell proliferation, stemness, angiogenesis, migration, invasiveness, extravasation, intravasation, metastasis, resistance to chemotherapy and in the microenvironment $(22,25,40-43)$.

In the present study, we firstly observed that WWOX protein was downregulated in osteosarcoma and that it regulated cell proliferation and EMT-associated gene expression (Fig. 7D). Moreover, WWOX overexpression suppressed the proliferation, migration and invasion of MG63 osteosarcoma cells. These results, along with the fact that the deletion of the WWOX gene and the frequent loss of its protein expression are detected in human osteosarcoma, and the tumor suppressor, WWOX, inhibits osteosarcoma metastasis by modulating RUNX2 function $(18,51)$, confirmed that WWOX is a tumor suppressor in osteosarcoma.

In line with the findings of previous studies demonstrating that miR-214-3p is an oncogene in various types of cancer (22,25,40-43), the silencing of miR-214-3p upregulated WWOX protein expression and inhibited the proliferation, migration and invasion of osteosarcoma cells. This finding may partly explain why WWOX protein is downregulated in osteosarcoma, since miR-214-3p is significantly upregulated in the disease. When we overexpressed WWOX in MG63 cells, it negatively regulated miR-214-3p. Thus, we concluded that the deregulation of microRNA-214-3p expression is involved in a negative feedback loop with WWOX in osteosarcoma. miR-10b has been proposed as an oncogene in various types of cancer (52-54). We found that WWOX significantly suppressed miR-10b expression in MG63 cells, although the role of miR-10b has not been reported in the disease, at least to the best of our knowledge.

In conclusion, the deregulation of microRNA-214-3p expression is involved in a negative feedback loop with WWOX in osteosarcoma, indicating that miR-214-3p has potential basic and clinical implications. On the one hand, WWOX may be a powerful osteosarcoma suppressor by exerting inhibitory effects on cell proliferation, migration and invasion, and the pharmacological restoration of WWOX may represent a promising therapeutic strategy. On the other hand, miR-214-3p as an oncogene is also associated with the pathogenesis and progression of osteosarcoma, and the targeting of miR-214-3p may also be apotential therapeutic strategy for patients with osteosarcoma.

\section{References}

1. Geller DS and Gorlick R: Osteosarcoma: A review of diagnosis, management, and treatment strategies. Clin Adv Hematol Oncol 8: 705-718, 2010.

2. Marina N, Gebhardt M, Teot L and Gorlick R: Biology and therapeutic advances for pediatric osteosarcoma. Oncologist 9: 422-441, 2004.

3. Wada T, Isu K, Takeda N, Usui M, Ishii S and Yamawaki S: A preliminary report of neoadjuvant chemotherapy NSH-7 study in osteosarcoma: Preoperative salvage chemotherapy based on clinical tumor response and the use of granulocyte colonystimulating factor. Oncology 53: 221-227, 1996.

4. Bednarek AK, Laflin KJ, Daniel RL, Liao Q, Hawkins KA and Aldaz CM: WWOX, a novel WW domain-containing protein mapping to human chromosome 16q23.3-24.1, a region frequently affected in breast cancer. Cancer Res 60: 2140-2145, 2000.

5. Ried K, Finnis M, Hobson L, Mangelsdorf M, Dayan S, Nancarrow JK, Woollatt E, Kremmidiotis G, Gardner A, Venter D, et al: Common chromosomal fragile site FRA16D sequence: Identification of the FOR gene spanning FRA16D and homozygous deletions and translocation breakpoints in cancer cells. Hum Mol Genet 9: 1651-1663, 2000.

6. Chang NS, Pratt N, Heath J, Schultz L, Sleve D, Carey GB and Zevotek N: Hyaluronidase induction of a WW domain-containing oxidoreductase that enhances tumor necrosis factor cytotoxicity. J Biol Chem 276: 3361-3370, 2001.

7. Paige AJ, Taylor KJ, Taylor C, Hillier SG, Farrington S, Scott D, Porteous DJ, Smyth JF, Gabra H and Watson JE: WWOX: A candidate tumor suppressor gene involved in multiple tumor types. Proc Natl Acad Sci USA 98: 11417-11422, 2001.

8. Kuroki T, Trapasso F, Shiraishi T, Alder H, Mimori K, Mori M and Croce CM: Genetic alterations of the tumor suppressor gene WWOX in esophageal squamous cell carcinoma. Cancer Res 62: 2258-2260, 2002.

9. Driouch K, Prydz H, Monese R, Johansen H, Lidereau R and Frengen E: Alternative transcripts of the candidate tumor suppressor gene, WWOX, are expressed at high levels in human breast tumors. Oncogene 21: 1832-1840, 2002.

10. Yendamuri S, Kuroki T, Trapasso F, Henry AC, Dumon KR, Huebner K, Williams NN, Kaiser LR and Croce CM: WW domain containing oxidoreductase gene expression is altered in non-small cell lung cancer. Cancer Res 63: 878-881, 2003.

11. Aqeilan RI, Kuroki T, Pekarsky Y, Albagha O, Trapasso F, Baffa R, Huebner K, Edmonds P and Croce CM: Loss of WWOX expression in gastric carcinoma. Clin Cancer Res 10: 3053-3058, 2004.

12. Kuroki T, Yendamuri S, Trapasso F, Matsuyama A, Aqeilan RI, Alder H, Rattan S, Cesari R, Nolli ML, Williams NN, et al: The tumor suppressor gene WWOX at FRA16D is involved in pancreatic carcinogenesis. Clin Cancer Res 10: 2459-2465, 2004.

13. Park SW, Ludes-Meyers J, Zimonjic DB, Durkin ME, Popescu NC and Aldaz CM: Frequent downregulation and loss of WWOX gene expression in human hepatocellular carcinoma. Br J Cancer 91: 753-759, 2004.

14. Pimenta FJ, Gomes DA, Perdigão PF, Barbosa AA, Romano-Silva MA, Gomez MV, Aldaz CM, De Marco L and Gomez RS: Characterization of the tumor suppressor gene WWOX in primary human oral squamous cell carcinomas. Int J Cancer 118: 1154-1158, 2006.

15. Bednarek AK, Keck-Waggoner CL, Daniel RL, Laflin KJ, Bergsagel PL, Kiguchi K, Brenner AJ and Aldaz CM: WWOX, the FRA16D gene, behaves as a suppressor of tumor growth. Cancer Res 61: 8068-8073, 2001. 
16. Fabbri M, Iliopoulos D, Trapasso F, Aqeilan RI, Cimmino A, Zanesi N, Yendamuri S, Han SY, Amadori D, Huebner K and Croce CM: WWOX gene restoration prevents lung cancer growth in vitro and in vivo. Proc Natl Acad Sci USA 102: 15611-15616, 2005.

17. Schirmer MA, Lüske CM, Roppel S, Schaudinn A, Zimmer C, Pflüger R, Haubrock M, Rapp J, Güngör C, Bockhorn M, et al: Relevance of Sp binding site polymorphism in WWOX for treatment outcome in pancreatic cancer. J Natl Cancer Inst 108: djv387, 2016

18. Del Mare S and Aqeilan RI: Tumor Suppressor WWOX inhibits osteosarcoma metastasis by modulating RUNX2 function. Sci Rep 5: 12959, 2015.

19. Calin GA and Croce CM: MicroRNA-cancer connection: The beginning of a new tale. Cancer Res 66: 7390-7394, 2006.

20. Esquela-Kerscher A and Slack FJ: Oncomirs - microRNAs with a role in cancer. Nat Rev Cancer 6: 259-269, 2006.

21. McManus MT: MicroRNAs and cancer. Semin Cancer Biol 13: 253-258, 2003

22. Xu CX, Xu M, Tan L, Yang H, Permuth-Wey J, Kruk PA Wenham RM, Nicosia SV, Lancaster JM, Sellers TA and Cheng JQ: MicroRNA miR-214 regulates ovarian cancer cell stemness by targeting p53/Nanog. J Biol Chem 287: 34970-34978, 2012.

23. Li X, Zhang Y, Zhang H, Liu X, Gong T, Li M, Sun L, Ji G, Shi Y, Han Z, et al: miRNA-223 promotes gastric cancer invasion and metastasis by targeting tumor suppressor EPB41L3. Mol Cancer Res 9: 824-833, 2011.

24. Zhang XJ, Ye H, Zeng CW, He B, Zhang $\mathrm{H}$ and Chen YQ: Dysregulation of miR-15a and miR-214 in human pancreatic cancer. J Hematol Oncol 3: 46, 2010.

25. Wang Z, Cai H, Lin L, Tang M and Cai H: Upregulated expression of microRNA-214 is linked to tumor progression and adverse prognosis in pediatric osteosarcoma. Pediatr Blood Cancer 61: 206-210, 2014

26. $\mathrm{Xu} \mathrm{Z}$ and Wang T: miR-214 promotes the proliferation and invasion of osteosarcoma cells through direct suppression of LZTS1. Biochem Biophys Res Commun 449: 190-195, 2014.

27. Lombaerts M, van Wezel T, Philippo K, Dierssen JW, Zimmerman RM, Oosting J, van Eijk R, Eilers PH, van de Water B, Cornelisse CJ and Cleton-Jansen AM: E-cadherin transcriptional downregulation by promoter methylation but not mutation is related to epithelial-to-mesenchymal transition in breast cancer cell lines. Br J Cancer 94: 661-671, 2006.

28. Shen A, Zhang Y, Yang H, Xu R and Huang G: Overexpression of ZEB1 relates to metastasis and invasion in osteosarcoma. J Surg Oncol 105: 830-834, 2012.

29. Cheng CW, Wang HW, Chang CW, Chu HW, Chen CY, Yu JC, Chao JI, Liu HF, Ding SL, Shen CY: MicroRNA-30a inhibits cell migration and invasion by downregulating vimentin expression and is a potential prognostic marker in breast cancer. Breast Cancer Res Treat 134: 1081-1093, 2012.

30. Gilles C, Polette M, Zahm JM, Tournier JM, Volders L, Foidart JM and Birembaut P: Vimentin contributes to human mammary epithelial cell migration. J Cell Sci 112: 4615-4625, 1999.

31. Uygur B and Wu WS: SLUG promotes prostate cancer cell migration and invasion via CXCR4/CXCL12 axis. Mol Cancer 10: 139, 2011.

32. Cheng GZ, Chan J, Wang Q, Zhang W, Sun CD and Wang LH: Twist transcriptionally up-regulates AKT2 in breast cancer cells leading to increased migration, invasion, and resistance to paclitaxel. Cancer Res 67: 1979-1987, 2007.

33. Burk U, Schubert J, Wellner U, Schmalhofer O, Vincan E, Spaderna $S$ and Brabletz T: A reciprocal repression between ZEB1 and members of the miR-200 family promotes EMT and invasion in cancer cells. EMBO Rep 9: 582-589, 2008.

34. Lee RC, Feinbaum RL and Ambros V: The C. elegans heterochronic gene lin-4 encodes small RNAs with antisense complementarity to lin-14. Cell 75: 843-854, 1993.

35. Pasquinelli AE, Reinhart BJ, Slack F, Martindale MQ, Kuroda MI, Maller B, Hayward DC, Ball EE, Degnan B, Müller P, et al: Conservation of the sequence and temporal expression of let-7 heterochronic regulatory RNA. Nature 408: 86-89, 2000.
36. He L, He X, Lim LP, de Stanchina E, Xuan Z, Liang Y, Xue W, Zender L, Magnus J, Ridzon D, et al: A microRNA component of the p53 tumour suppressor network. Nature 447: 1130-1134, 2007.

37. Novakova J, Slaby O, Vyzula R and Michalek J: MicroRNA involvement in glioblastoma pathogenesis. Biochem Biophys Res Commun 386: 1-5, 2009.

38. Sasayama T, Nishihara M, Kondoh T, Hosoda K and Kohmura E: MicroRNA-10b is overexpressed in malignant glioma and associated with tumor invasive factors, uPAR and RhoC. Int J Cancer 125: 1407-1413, 2009.

39. Guessous F, Zhang Y, Kofman A, Catania A, Li Y, Schiff D, Purow B and Abounader R: microRNA-34a is tumor suppressive in brain tumors and glioma stem cells. Cell Cycle 9: 1031-1036, 2010.

40. Yang TS, Yang XH, Wang XD, Wang YL, Zhou B and Song ZS: MiR-214 regulate gastric cancer cell proliferation, migration and invasion by targeting PTEN. Cancer Cell Int 13: 68, 2013.

41. Lu S, Gao Y, Huang X and Wang X: Cantharidin exerts antihepatocellular carcinoma by miR-214 modulating macrophage polarization. Int J Biol Sci 10: 415-425, 2014.

42. Wang YS, Wang YH, Xia HP, Zhou SW, Schmid-Bindert G and Zhou CC: MicroRNA-214 regulates the acquired resistance to gefitinib via the PTEN/AKT pathway in EGFR-mutant cell lines. Asian Pac J Cancer Prev 13: 255-260, 2012.

43. Xia H, Ooi LL and Hui KM: MiR-214 targets $\beta$-catenin pathway to suppress invasion, stem-like traits and recurrence of human hepatocellular carcinoma. PLoS One 7: e44206, 2012.

44. Zhang W, Qian JX, Yi HL, Yang ZD, Wang CF, Chen JY, Wei XZ, Fu Q and Ma H: The microRNA-29 plays a central role in osteosarcoma pathogenesis and progression. Mol Biol (Mosk) 46: 622-627, 2012.

45. Bao YP, Yi Y, Peng LL, Fang J, Liu KB, Li WZ and Luo HS: Roles of microRNA-206 in osteosarcoma pathogenesis and progression. Asian Pac J Cancer Prev 14: 3751-3755, 2013.

46. Zhou L, Park J, Jang KY, Park HS, Wagle S, Yang KH, Lee KB, Park BH and Kim JR: The overexpression of BAMBI and its involvement in the growth and invasion of human osteosarcoma cells. Oncol Rep 30: 1315-1322, 2013.

47. Jones KB, Salah Z, Del Mare S, Galasso M, Gaudio E, Nuovo GJ, Lovat F, LeBlanc K, Palatini J, Randall RL, et al: miRNA signatures associate with pathogenesis and progression of osteosarcoma. Cancer Res 72: 1865-1877, 2012.

48. Penna E, Orso F, Cimino D, Vercellino I, Grassi E, Quaglino E, Turco E and Taverna D: miR-214 coordinates melanoma progression by upregulating ALCAM through TFAP2 and miR-148b downmodulation. Cancer Res 73: 4098-4111, 2013.

49. Derfoul A, Juan AH, Difilippantonio MJ, Palanisamy N, Ried T and Sartorelli V: Decreased microRNA-214 levels in breast cancer cells coincides with increased cell proliferation, invasion and accumulation of the Polycomb Ezh2 methyltransferase. Carcinogenesis 32: 1607-1614, 2011.

50. Juan AH, Kumar RM, Marx JG, Young RA and Sartorelli V: MiR-14-dependent regulation of the polycomb protein Ezh2 in skeletal muscle and embryonic stem cells. Mol Cell 36: 61-74, 2009.

51. Yang J, Cogdell D, Yang D, Hu L, Li H, Zheng H, Du X, Pang Y, Trent J, Chen K and Zhang W: Deletion of the WWOX gene and frequent loss of its protein expression in human osteosarcoma. Cancer Lett 291: 31-38, 2010.

52. Ma L, Teruya-Feldstein $\mathbf{J}$ and Weinberg RA: Tumour invasion and metastasis initiated by microRNA-10b in breast cancer. Nature 449: 682-688, 2007.

53. Liu Z, Zhu J, Cao H, Ren H and Fang X: miR-10b promotes cell invasion through RhoC-AKT signaling pathway by targeting HOXD10 in gastric cancer. Int J Oncol 40: 1553-1560, 2012.

54. Guessous F, Alvarado-Velez M, Marcinkiewicz L, Zhang Y, Kim J, Heister S, Kefas B, Godlewski J, Schiff D, Purow B and Abounader R: Oncogenic effects of miR-10b in glioblastoma stem cells. J Neurooncol 112: 153-163, 2013. 